\section{The American Association for Thoracic Surgery Volunteer Leadership and Senior Staff Disclosure Statement}

To best serve the interests of the specialty and its practitioners, the American Association for Thoracic Surgery requires the volunteer leadership, including its editors, associate editors, and section editors, to publicly disclose the existence and nature of any financial or other relationships that might influence, or appear to influence, their actions. In keeping with this policy, the following disclosures have been made by the editors and editorial board members responsible for making decisions about manuscripts submitted to and published in the official publications of the Association:

\begin{tabular}{ll}
\hline Lawrence Cohn & JTCVS Editor \\
Fred Crawford & Op Techs Editor \\
Harvey Pass & Seminars Editor
\end{tabular}

Todd Rosengart Joseph Dearani Jennifer Lawton Niv Ad

Sary Aranki
Erle Austin
Carl Backer
Vinay Badhwar
Leora Balsam
Munir Boodhwani
Michael Borger
Scott Bradley
Christopher Caldarone
Duke Cameron
Andrea Carpenter
Thierry-Pierre Carrel
Robert Cerfolio

Fred Chen

Yolanda Colson

Michael D'Ambra

Hiroshi Date William DeCampli Chad Denlinger John Elefteriades

James Fann Victor Ferraris Daniel Fitzgerald Raja Flores Charles Fraser J. William Gaynor

Donald Glower Alden Harken Shen-shou Hu
Seminars Editor

Pediatrics Annual Editor/JTCVS Editorial Board TSN Editor

JTCVS Editorial Board

JTCVS Editorial Board JTCVS Editorial Board JTCVS Editorial Board JTCVS Editorial Board JTCVS Editorial Board JTCVS Editorial Board JTCVS Editorial Board

JTCVS Editorial Board JTCVS Associate Editor JTCVS Editorial Board JTCVS Editorial Board JTCVS Editorial Board JTCVS Editorial Board

JTCVS Editorial Board JTCVS Editorial Board JTCVS Editorial Board

JTCVS Editorial Board JTCVS Editorial Board JTCVS Editorial Board JTCVS Editorial Board

JTCVS Editorial Board JTCVS Editorial Board JTCVS Editorial Board JTCVS Editorial Board JTCVS Editorial Board JTCVS Editorial Board

JTCVS Editorial Board JTCVS Editorial Board JTCVS Editorial Board
Nothing to Disclose

Medronic, Stock; Johnson and Johnson, Stock

Champions Oncology, Advisory Board; General Electric, Advisory Board; Intergrated Diagnostics, Research Collaborator; National Cancer Institute, Research Funding; Belluck and Fox, Philanthropy; Levi, Phillips, and Konigsberg, Philanthropy; Pinpoint Genomics, Advisory Board; Celera, Research Collaborator; Somalogic, Research Collaborator; GSK, Advisory Panel; Quest, Advisory Board; Transgenomics, Research Funding

Nothing to Disclose

Nothing to Disclose

Nothing to Disclose

Medtronic, Consultant; Atricure, Consultant; Left Atrial Appendage Occluder LLC, Part Owner

Nothing to Disclose

Nothing to Disclose

Nothing to Disclose

Nothing to Disclose

Nothing to Disclose

Nothing to Disclose

Edwards Lifesciences, Speakers; Medtronic, Speaker; St. Jude Medical, Speaker

Nothing to Disclose

CellAegis Inc, Ownership;

Nothing to Disclose

Nothing to Disclose

Nothing to Disclose

Intuitive Surgical, Faculty; Pfizer, Research Grant; Neomend, Research Grant; Precision Therapeutics, Research Grant

Nothing to Disclose

Nothing to Disclose

PeriCor Therapeutics; Board of Directors; D'Ambra Technologies LLC, Partner; Scanlan Instruments; Consultant

Nothing to Disclose

Nothing to Disclose

Nothing to Disclose

Covidien, Consultant; Datascope, Consultant; CoolSpine, Medical Director; Medtronic, PI

Apica Cardiovascular Limited, Consultant; Twelve Inc, Consultant

Haemonetics, Advisory Board

Nothing to Disclose

Nothing to Disclose

Nothing to Disclose

Johnson and Johnson, Stock; Bristol Myers Squibb, Stock; Medtronic, Stock; Pfizer, Stock; General Electric, Bonds and Stock; Lilly; Stock; Merck, Stock; Dow, Stock; Dupont, Stock

Nothing to Disclose

Nothing to Disclose

Nothing to Disclose 


\begin{tabular}{|c|c|c|}
\hline Kirk Kanter & JTCVS Editorial Board & Nothing to Disclose \\
\hline Ben Kozower & JTCVS Editorial Board & Nothing to Disclose \\
\hline Nevin Katz & JTCVS Associate Editor & Nothing to Disclose \\
\hline Irving Kron & JTCVS Editorial Board & Nothing to Disclose \\
\hline Paul Kurlansky & JTCVS Editorial Board & Nothing to Disclose \\
\hline Jennifer Lawton & JTCVS Editorial Board & Nothing to Disclose \\
\hline Harold Lazar & JTCVS Editorial Board & Eli Lilly Inc, Research Grant \\
\hline Michael Liptay & JTCVS Editorial Board & Covidien, Proctor and Course Director \\
\hline Audrey Marshall & JTCVS Editorial Board & Nothing to Disclose \\
\hline Patrick McCarthy & JTCVS Editorial Board & $\begin{array}{l}\text { Edwards Lifesciences LLC, Intellectual Property and Consulting; Cardious } \\
\text { Inc, Consultant; Baxter, Advisory Board; Abbott, Consultant; Micardia, } \\
\text { Consultant; Direct Flow; Travel }\end{array}$ \\
\hline P. Michael McFadden & JTCVS Editorial Board & Nothing to Disclose \\
\hline Carlos Mestres & JTCVS Editorial Board & $\begin{array}{l}\text { Novartis Pharma, Advisory Board; Edwards Lifescience, Consultant; } \\
\text { Symetis SA, Advisory Board }\end{array}$ \\
\hline Marc Moon & JTCVS Editorial Board & Nothing to Disclose \\
\hline Christine Mora-Mangano & JTCVS Editorial Board & Pericor Inc, Owner; ATGC, Owner \\
\hline Sudish Murthy & JTCVS Editorial Board & Nothing to Disclose \\
\hline Katie Nason & JTCVS Editorial Board & Nothing to Disclose \\
\hline Thomas Ng & JTCVS Editorial Board & Nothing to Disclose \\
\hline Takashi Nishimura & JTCVS Editorial Board & Nothing to Disclose \\
\hline Soon Park & JTCVS Editorial Board & Nothing to Disclose \\
\hline Frank Pigula & JTCVS Editorial Board & Nothing to Disclose \\
\hline Hans-Joachim Schaefers & JTCVS Editorial Board & Nothing to Disclose \\
\hline Oz Shapira & JTCVS Editorial Board & Nothing to Disclose \\
\hline Richard Shemin & JTCVS Editorial Board & Edwards Lifesciences, Consultant; AtriCure, Consultant \\
\hline Edward Soltesz & JTCVS Editorial Board & St. Jude Medical, Speaker; TransMedics, Steering Committee Member \\
\hline Rakesh Suri & JTCVS Editorial Board & $\begin{array}{l}\text { Edward Lifesciences, Research Grant; Sorin, Research Grant, PI, IP; St. Jude } \\
\text { Medical, Research Grant, Steering Committee }\end{array}$ \\
\hline Lars Svensson & JTCVS Editorial Board & Cardiosolutions; Posthorax; ValveXchange \\
\hline John Toole & JTCVS Editorial Board & Nothing to Disclose \\
\hline Dirk Van Raemdonck & JTCVS Editorial Board & Nothing to Disclose \\
\hline Federico Venuta & JTCVS Editorial Board & \\
\hline Jakob Vinten-Johansen & JTCVS Editorial Board & Somahlution Inc, Consultant \\
\hline Y. Joseph Woo & JTCVS Editorial Board & Nothing to Disclose \\
\hline Stephen Yang & JTCVS Editorial Board & Nothing to Disclose \\
\hline Richard Weisel & JTCVS Editorial Board & Nothing to Disclose \\
\hline William Williams & Associate Editor, Thoracic Surgery News & Nothing to Disclose \\
\hline Mara Antonoff & TSRA Associate Editor, Thoracic Surgery News & Nothing to Disclose \\
\hline Sanford Zeigler & TSRA Associate Editor, Thoracic Surgery News & Nothing to Disclose \\
\hline John Ikonomidis & JTCVS Associate Editor & Nothing to Disclose \\
\hline Hartzell Schaff & JTCVS Associate Editor & Nothing to Disclose \\
\hline Cary Akins & JTCVS Associate Editor & $\begin{array}{l}\text { Medtronic Inc, Licensed Patent for a Perfusion Cannula; Edwards } \\
\text { LifeSciences, Data Safety Monitoring Board Member }\end{array}$ \\
\hline Emile Bacha & JTCVS Associate Editor & Cormatrix, Scientific Advisory Board \\
\hline Frank Sellke & JTCVS Associate Editor & $\begin{array}{l}\text { Boehringer Ingelheim, DSMB; CSL Behring, Adjudication Committee for } \\
\text { Trial; Pfizer, Expert witness and consultant on Bextra/Celebrex litigation }\end{array}$ \\
\hline Eugene Blackstone & JTCVS Associate Editor & $\begin{array}{l}\text { Churchill-Livingstone Book Royalties, Author; Edwards Lifesciences, } \\
\text { PARTNER Publication Office (independent analysis of trial data }\end{array}$ \\
\hline Bryan Meyers & JTCVS Associate Editor & $\begin{array}{l}\text { Ethicon Endo-Surgery, Speaker, Teacher, Consultant on Educational Effort; } \\
\text { Varian, Speaker, Consultant }\end{array}$ \\
\hline Thomas Rice & JTCVS Associate Editor & Nothing to Disclose \\
\hline Ara Vaporciyan & JTCVS Associate Editor & Nothing to Disclose \\
\hline
\end{tabular}

